\title{
Direct Contacting of 2D Nanosheets by Metallic Nanoprobes ${ }^{\dagger}$
}

\author{
Filippo Giubileo ${ }^{1, *}$, Francesca Urban ${ }^{1,2}$, Alessandro Grillo ${ }^{1,2}$, Aniello Pelella ${ }^{1,2}$, Enver Faella ${ }^{2}$ \\ and Antonio Di Bartolomeo ${ }^{1,2}$
}

Citation: Giubileo, F.; Urban, F.; Grillo,

A.; Pelella, A..; Faella, E.; Di

Bartolomeo, A. Direct Contacting of 2D Nanosheets by Metallic Nanoprobes. Mater. Proc. 2021, 4, 16. https:// doi.org/10.3390/IOCN2020-07931

Academic Editors: Ana María Díez-Pascual and Guanying Chen

Published: 11 November 2020

Publisher's Note: MDPI stays neutral with regard to jurisdictional claims in published maps and institutional affiliations.

Copyright: $₫ 2020$ by the authors. Licensee MDPI, Basel, Switzerland. This article is an open access article distributed under the terms and conditions of the Creative Commons Attribution (CC BY) license (http://creativecommons.org/licenses /by/4.0/).
1 CNR-SPIN Salerno Via Giovanni Paolo II 132, 84084 Fisciano, Salerno, Italy; furban@unisa.it (F.U.); agrillo@unisa.it (A.G.); apelella@unisa.it (A.P.); adibartolomeo@unisa.it (A.D.B.)

2 Physics Department “E. R. Caianiello”, University of Salerno, Via Giovanni Paolo II 132, 84084 Fisciano, Salerno, Italy; efaella@unisa.it

* Correspondence: filippo.giubileo@spin.cnr.it

+ Presented at the 2nd International Online-Conference on Nanomaterials, 15-30 November 2020; Available online: https://iocn2020.sciforum.net/.

\begin{abstract}
We present a simple and fast methodology to realize metal contacts on two-dimensional nanosheets. In particular, we perform a complete characterization of the transport properties of $\mathrm{MoS}_{2}$ monolayer flakes on $\mathrm{SiO}_{2} / \mathrm{Si}$ substrates by using nano-manipulated metallic tips as metallic electrodes directly approached on the flake surface. We report detailed experimental investigation of transport properties and contact resistance in back-gated field effect transistor in which the Si substrate is used as the gate electrode. Moreover, profiting of the n-type conduction, as well as the high aspect ratio at the edge of the MoS2 flakes, we also explored the possibility of exploiting the material as a field emitter. Indeed, by retracting one of the metallic probes (the anode) from the sample surface, it has been possible to switch on a field-emitted current by applying a relatively low external electric field of few-tens of Volts for a cathode-anode separation distance below $1 \mu \mathrm{m}$. Experimental data are then analyzed in the framework of Fowler-Nordheim theory and its extension to the two-dimensional limit.
\end{abstract}

Keywords: two-dimensional materials; transition metal dichalcogenides; molybdenum disulfide; field-effect transistor; transport properties; field emission

\section{Introduction}

Molybdenum disulfide $\left(\mathrm{MoS}_{2}\right)$ is one of the most investigated transition-metal dichalcogenides (TMDs) for exploitation in next-generation two-dimensional (2D) devices, including field-effect transistors [1-4], solar cells [5], photodetectors [6,7], field emission devices [8-11], chemical or biological sensors [12,13], etc.

$\mathrm{MoS}_{2}$ has a crystal structure characterized by a hexagonal layer of Mo atoms between two layers of $S$ atoms. Layers are bonded together by van der Waals forces. MoS flakes can be fabricated either by mechanical exfoliation or chemical vapor deposition [14]. Bulk $\mathrm{MoS}_{2}$ has $1.2 \mathrm{eV}$ indirect bandgap, while mono-layer (1 L) and bilayer (2 L) MoS have 1.8 $\mathrm{eV}$ and $1.6 \mathrm{eV}$ indirect bandgap, respectively [15]. Consequently, both $1 \mathrm{~L}$ and $2 \mathrm{~L} \mathrm{MoS}_{2}$ can be used to realize field-effect transistors with high On/Off ratio and photoresponse [16]. On the other hand, carrier mobility is typically limited to few-tens $\mathrm{cm}^{2} \mathrm{~V}^{-1} \mathrm{~s}^{-1}$. Moreover, ohmic contacts (with low resistance) are crucial to improving device performance [17].

In this paper, we demonstrate a simple method of realizing electrical contacts on $\mathrm{MoS}_{2}$ flakes by using nanomanipulated metallic probes inside a scanning electron microscope (SEM). We show that this technique allows complete characterization of the back-gated field-effect transistor (FET), as well as checking the field emission properties of the $\mathrm{MoS}_{2}$ flake. 


\section{Materials and Methods}

The $\mathrm{MoS}_{2}$ flakes studied in this work have been grown on $\mathrm{Si} / \mathrm{SiO}_{2}$ substrates by means of a chemical vapour deposition technique, in which $S$ powder and a saturated ammonium heptamolybdate solution have been used as precursors. Few-layer $\mathrm{MoS}_{2}$ flakes have been characterized by micro-Raman spectroscopy $(\lambda=532 \mathrm{~nm})$. The experimental setup for electrical characterization is realized inside a SEM chamber (see Figure 1a) provided with two piezo-driven nano-manipulators for precise positioning (step resolution $\sim 5 \mathrm{~nm}$ ) of metallic probes (tungsten tips). A semiconductor parameter analyzer (Keithley 4200-SCS) is then used as a source-measurement unit, to apply bias up to $\pm 100 \mathrm{~V}$ and to measure current with resolution better than $0.1 \mathrm{pA}$. Electrical measurements are performed at room temperature and in high vacuum $\left(10^{-6} \mathrm{mbar}\right)$ after gently approaching the tungsten tips on the $\mathrm{MoS}_{2}$ flake (a real image taken inside the SEM chamber is shown in Figure 1b) and using the Si substrate as a back gate.
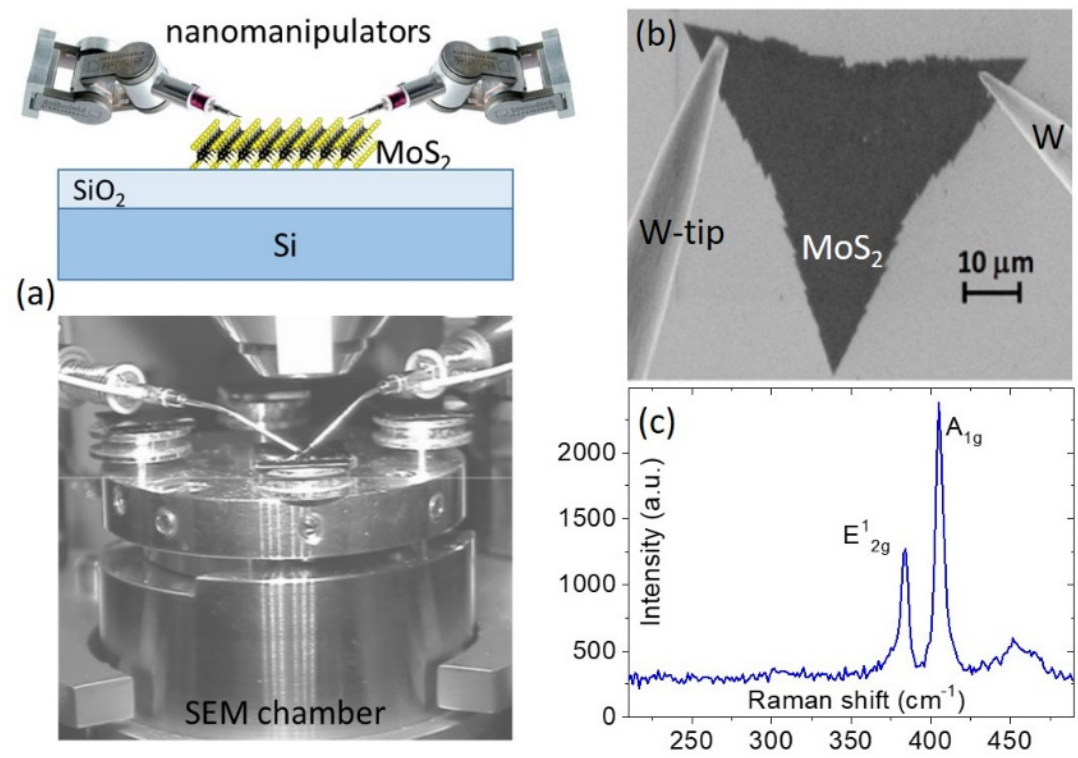

Figure 1. (a) Schematic and real image of the nanomanipulators contacting the $\mathrm{MoS}_{2}$ flake inside the SEM chamber. (b) SEM image of a contacted $\mathrm{MoS}_{2}$ flake. (c) Raman spectrum of MoS2 flake.

Micro-Raman analysis of the $\mathrm{MoS}_{2}$ flake has shown a spectrum (see Figure 1c) with two peaks corresponding to the $E_{2 g}^{1}$ and $A_{1 g}$ modes, separated by about $20 \mathrm{~cm}^{-1}$, indicating that the sample under investigation is a monolayer.

\section{Results and Discussion}

In Figure 2a, we report the output characteristics $\left(I_{d s}-V_{d s}\right)$ measured in the range of $\pm 0.5 \mathrm{~V}$ for different values of the gate voltage $\left(V_{g s}\right)$. We notice a slight rectification that can be explained as the result of asymmetric Schottky barriers forming at the tungsten/MoS interfaces [3]. By varying the distance between the two tungsten tips, we can modulate the channel length of the FET, thus realizing an experiment based on the Transfer Length Method (TLM) $[18,19]$ to evaluate the contact resistance at the tungsten/MoS $\mathrm{M}_{2}$ interface. In Figure $2 \mathrm{~b}$, we show the measured total resistance $R_{\text {tot }}$ versus $d$, with $R_{\text {tot }}=2 R_{c}+\frac{R_{s}}{W} d$, where $R_{c}$ is the contact resistance, $R_{s}$ is the MoS2 sheet resistance, $\mathrm{W}$ is the channel width (assumed to be equal to the tip diameter, $200 \mathrm{~nm}$ ), and $d$ is the channel length, i.e., the separation between the two tips. Experimental data have linear behavior, from which specific area contact resistivity and sheet resistance can be evaluated as $\rho_{c} \approx$ $4 \times 10^{-2} \Omega \mathrm{cm}^{2}$ and $R_{s} \approx 10^{8} \Omega / \square$ from the intercept and the slope of the linear fit, respectively. 
The transfer characteristics $\left(I_{d s}-V_{g s}\right)$ reported in Figure 2c have been measured for different gate voltage ranges up to $\pm 60 \mathrm{~V}$, with $V_{d s}=-5 \mathrm{~V}$, and by positioning the tungsten tips at separation of $13 \mu \mathrm{m}$. The device has n-type behavior, with a threshold voltage of about $-10 \mathrm{~V}$, and it can be explained in terms of chemisorption of oxygen on $\mathrm{MoS}_{2}$ or sulphur vacancies [20-22].
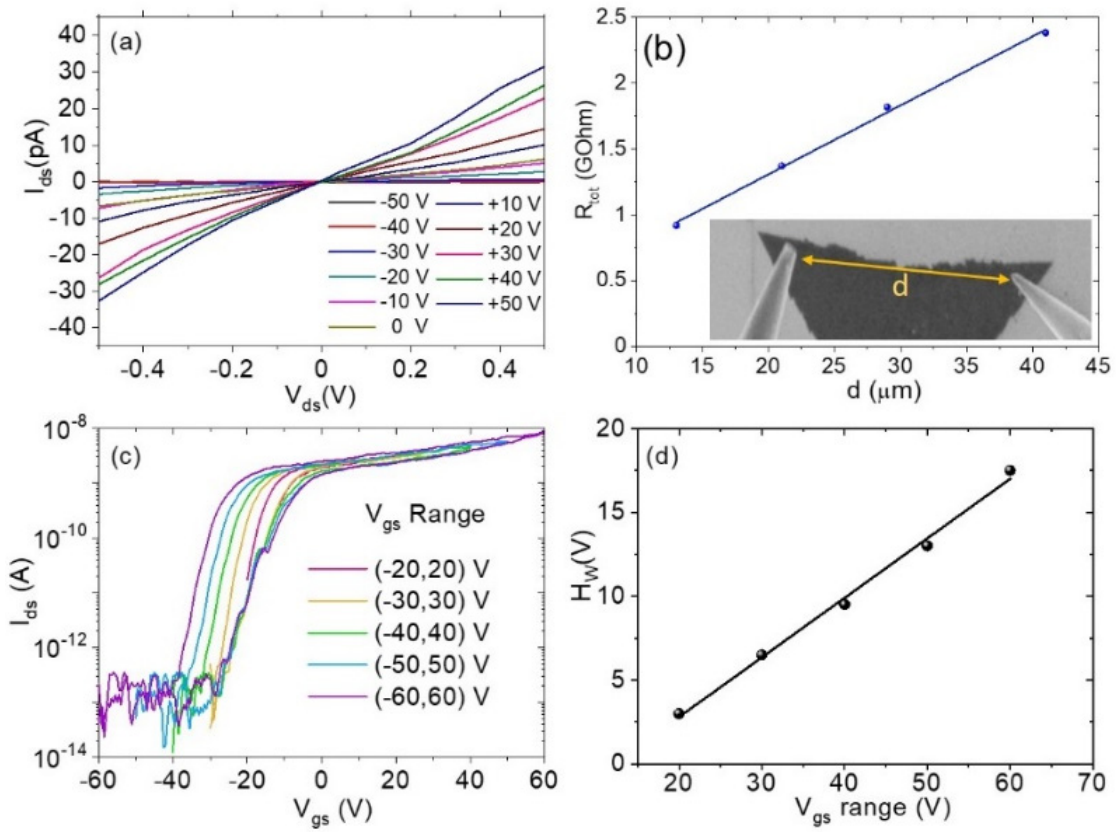

Figure 2. (a) Output characteristics $\left(I_{d s}-V_{d s}\right)$ measured for different gate voltage values. (b) $R_{\text {tot }}$ vs $d$ plot and linear fit. The inset show how $d$ is measured. (c) Transfer characteristics $\left(I_{d s}-V_{g s}\right)$ measured for different gate voltage ranges. (d) Linear dependence of the hysteresis width $\mathrm{Hw}$ as a function of $V_{g s}$.

From the transfer characteristic measured in the range $\pm 50 \mathrm{~V}$, we have estimated the on/off ratio as $\sim 10^{5}$, a subthreshold swing of $S S \approx 4 \frac{V}{\text { decade }}$, and a mobility of $\mu=$ $1 \mathrm{~cm}^{2} \mathrm{~V}^{-1} \mathrm{~s}^{-1}$, a value within the typical range $\left(0.02-100 \mathrm{~cm}^{2} \mathrm{~V}^{-1} \mathrm{~s}^{-1}\right)$ reported for $\mathrm{MoS}_{2}-$ based FETs on $\mathrm{SiO}_{2}[23,24]$. The low mobility can be attributed to the high contact resistance and to high defects or traps density [25].

In Figure 3c, we show the transfer characteristics measured by sweeping the gate voltage different ranges, from $\pm 20 \mathrm{~V}$ up to $\pm 60 \mathrm{~V}$. The curves have a clear hysteresis that we explain as being caused by negative charge trapping [20]. We observe that the hysteresis width $\left(H_{W}\right)$, estimated at $I_{d s}=0.1 \mathrm{nA}$, has linear dependence on the $V_{g s}$ sweeping range (See Figure $3 \mathrm{~d}$ ). This behavior can be ascribed to the trapping process driven by the gate voltage and the effects on the $\mathrm{MoS}_{2} / \mathrm{Si}$-substrate capacitor.

Finally, we also investigated the field emission (FE) properties of the MoS flake, profiting of the n-type conduction and the high aspect ratio of the flake side. By retracting the tip-anode at a distance $h=900 \mathrm{~nm}$ from the MoS 2 edge, we can measure the current emitted from the flake under the application of an external electric field (Figure 3a). More precisely, we applied a voltage bias of up to $120 \mathrm{~V}$ on the anode, and we measured the current emitted from the flake (cathode) with a resolution better that $0.1 \mathrm{pA}$. The current-voltage $\left(I_{d s}-V_{d s}\right)$ curves have been measured at fixed cathode-anode separation $h$ and for two different values of gate voltage. 
(a)
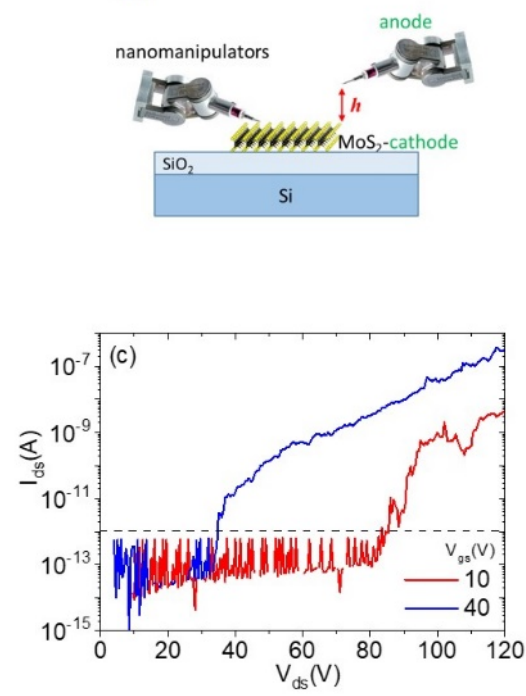
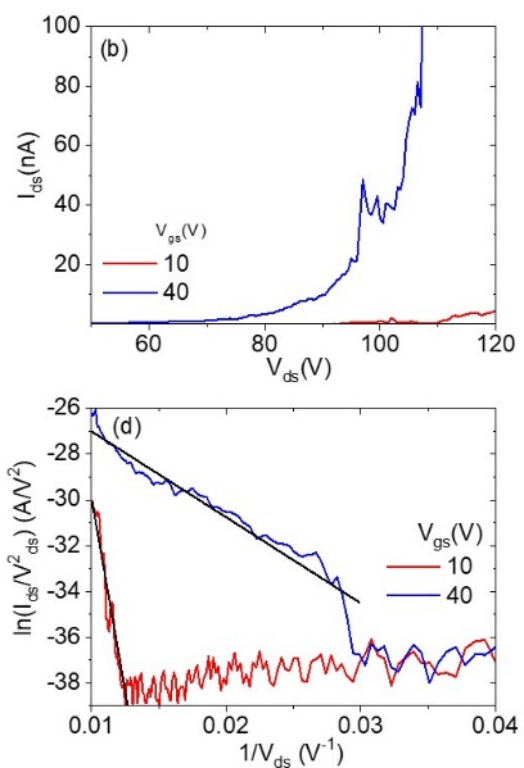

Figure 3. (a) Schematic of field emission setup. (b) $I_{d s}-V_{d s}$ field emission curves on a linear scale, measured for two different gate voltages. (c) Same $I_{d s}-V_{d s}$ field emission curves, reported on a logarithmic scale. (d) Fowler-Nordheim plots and linear fittings.

The FE characteristics have been measured by applying a bias voltage on the anode up to $+120 \mathrm{~V}$, by keeping a fixed gate voltage of $10 \mathrm{~V}$ and $40 \mathrm{~V}$, respectively. The measured curves are reported on a linear scale (Figure $3 b$ ) and on a logarithmic scale (Figure $3 c$ ). Interestingly, we observe that the FE current is larger for $V_{g s}=40 \mathrm{~V}$, suggesting that the gate voltage increases the n-doping of the $\mathrm{MoS}_{2}$ flake [26].

We analyzed the FE curves in the framework of the Fowler-Nordheim (FN) theory [27], for which the FE current is expressed as

$$
I_{d s}=A \frac{\left(\beta V_{d s} / h\right)^{2}}{\Phi} S \cdot \exp \left(-B \frac{\Phi^{\frac{3}{2}}}{\left(\beta V_{d s} / h\right)}\right),
$$

where $A=1.54 \times 10^{-6} \mathrm{~A} \mathrm{~V}^{-2} \mathrm{eV}$ and $B=6.83 \times 10^{7} \mathrm{~V} \mathrm{~cm}^{-1} \mathrm{eV}^{-3 / 2}, \Phi$ is the work function of the emitter, $S$ is the emitting surface area, and $\beta$ is the field enhancement factor. Accordingly, for FE curves, it is expected that $\ln \left(I_{d s} / V_{d s}^{2}\right)$ versus $1 / V$ is linear (FN plot), and $\beta$ can be evaluated from its slope.

In Figure 3d, we report the FN plots that demonstrate the FE nature of the measured current. For $V_{g s}=40 \mathrm{~V}$, we found a turn-on field $E_{o n}=40 \mathrm{~V} \mathrm{um}^{-1}$ (defined as the field to obtain a FE current of $1 \mathrm{pA}$ ) and $\beta \approx 200$.

\section{Conclusions}

We demonstrate a simple and fast methodology to realize metal contacts on twodimensional nanosheets by gently approaching nanomanipulated tungsten tips inside a scanning electron microscope. We contacted a $\mathrm{MoS}_{2}$ monolayer to form a back-gated FET, and we performed complete electrical characterization, reporting specific area contact resistivity of $4 \times 10^{-2} \Omega \mathrm{cm}^{2}$, sheet resistance of $10^{8} \Omega / \square$, on/off ratio of $10^{5}$, subthreshold swing of $4 \mathrm{~V} /$ decade, and mobility of $1 \mathrm{~cm}^{2} \mathrm{~V}^{-1} \mathrm{~s}^{-1}$. Finally, by retracting the tip-anode, we performed field emission characterization of the $\mathrm{MoS}_{2}$ flake, reporting that the FE current can be modulated by the gate bias. 
Data Availability Statement: Data available on request.

Conflicts of Interest: The authors declare no conflict of interest.

\section{References}

1. Tong, X.; Ashalley, E.; Lin, F.; Li, H.; Wang, Z.M. Advances in MoS2-Based Field Effect Transistors (FETs). Nano-Micro Lett. 2015, 7, 203-218, doi:10.1007/s40820-015-0034-8.

2. Seo, D.; Lee, D.Y.; Kwon, J.; Lee, J.J.; Taniguchi, T.; Watanabe, K.; Lee, G.-H.; Kim, K.S.; Hone, J.; Kim, Y.D.; et al. Highperformance monolayer MoS2 field-effect transistor with large-scale nitrogen-doped graphene electrodes for Ohmic contact. Appl. Phys. Lett. 2019, 115, 012104, doi:10.1063/1.5094682.

3. Di Bartolomeo, A.; Grillo, A.; Urban, F.; Iemmo, L.; Giubileo, F.; Luongo, G.; Amato, G.; Croin, L.; Sun, L.; Liang, S.-J.; et al. Asymmetric Schottky Contacts in Bilayer MoS2 Field Effect Transistors. Adv. Funct. Mater. 2018, 28, 1800657, doi:10.1002/adfm.201800657.

4. Divya Bharathi, N.; Sivasankaran, K. Research progress and challenges of two dimensional MoS2 field effect transistors. J. Semicond. 2018, 39, 104002, doi:10.1088/1674-4926/39/10/104002.

5. Hao, L.; Liu, Y.; Gao, W.; Han, Z.; Xue, Q.; Zeng, H.; Wu, Z.; Zhu, J.; Zhang, W. Electrical and photovoltaic characteristics of $\mathrm{MoS}_{2} /$ Si $p$ - $n$ junctions. J. Appl. Phys. 2015, 117, 114502, doi:10.1063/1.4915951.

6. Lopez-Sanchez, O.; Lembke, D.; Kayci, M.; Radenovic, A.; Kis, A. Ultrasensitive photodetectors based on monolayer MoS2. Nat. Nanotechnol. 2013, 8, 497-501, doi:10.1038/nnano.2013.100.

7. Di Bartolomeo, A.; Genovese, L.; Foller, T.; Giubileo, F.; Luongo, G.; Croin, L.; Liang, S.-J.; Ang, L.K.; Schleberger, M. Electrical transport and persistent photoconductivity in monolayer MoS 2 phototransistors. Nanotechnology 2017, 28, 214002, doi:10.1088/1361-6528/aa6d98.

8. Fu, H.; Yu, K.; Li, H.; Li, J.; Guo, B.; Tan, Y.; Song, C.; Zhu, Z. Enhanced field emission and photocatalytic performance of MoS 2 titania nanoheterojunctions via two synthetic approaches. Dalton Trans. 2015, 44, 1664-1672, doi:10.1039/C4DT03035D.

9. Kashid, R.V.; Late, D.J.; Chou, S.S.; Huang, Y.-K.; De, M.; Joag, D.S.; More, M.A.; Dravid, V.P. Enhanced Field-Emission Behavior of Layered MoS 2 Sheets. Small 2013, 9, 2730-2734, doi:10.1002/smll.201300002.

10. Giubileo, F.; Grillo, A.; Passacantando, M.; Urban, F.; Iemmo, L.; Luongo, G.; Pelella, A.; Loveridge, M.; Lozzi, L.; Di Bartolomeo, A. Field Emission Characterization of MoS2 Nanoflowers. Nanomaterials 2019, 9, 717, doi:10.3390/nano9050717.

11. Giubileo, F.; Iemmo, L.; Passacantando, M.; Urban, F.; Luongo, G.; Sun, L.; Amato, G.; Enrico, E.; Di Bartolomeo, A. Effect of Electron Irradiation on the Transport and Field Emission Properties of Few-Layer MoS 2 Field-Effect Transistors. J. Phys. Chem. C 2019, 123, 1454-1461, doi:10.1021/acs.jpcc.8b09089.

12. Li, P.; Zhang, D.; Sun, Y.; Chang, H.; Liu, J.; Yin, N. Towards intrinsic MoS 2 devices for high performance arsenite sensing. Appl. Phys. Lett. 2016, 109, 063110, doi:10.1063/1.4960967.

13. Yan, L.; Shi, H.; Sui, X.; Deng, Z.; Gao, L. MoS 2 -DNA and MoS 2 based sensors. RSC Adv. 2017, 7, 23573-23582, doi:10.1039/C7RA02649H.

14. Sun, J.; Li, X.; Guo, W.; Zhao, M.; Fan, X.; Dong, Y.; Xu, C.; Deng, J.; Fu, Y. Synthesis Methods of Two-Dimensional MoS2: A Brief Review. Crystals 2017, 7, 198, doi:10.3390/cryst7070198.

15. Mak, K.F.; Lee, C.; Hone, J.; Shan, J.; Heinz, T.F. Atomically Thin MoS 2: A New Direct-Gap Semiconductor. Phys. Rev. Lett. 2010, 105, doi:10.1103/PhysRevLett.105.136805.

16. Lee, Y.T.; Kang, J.-H.; Kwak, K.; Ahn, J.; Choi, H.T.; Ju, B.-K.; Shokouh, S.H.; Im, S.; Park, M.-C.; Hwang, D.K. High-Performance 2D MoS2 Phototransistor for Photo Logic Gate and Image Sensor. ACS Photonics 2018, 5, 4745-4750, doi:10.1021/acsphotonics.8b01049.

17. Giubileo, F.; Di Bartolomeo, A. The role of contact resistance in graphene field-effect devices. Prog. Surf. Sci. 2017, 92, 143-175, doi:10.1016/j.progsurf.2017.05.002.

18. Giubileo, F.; Di Bartolomeo, A.; Martucciello, N.; Romeo, F.; Iemmo, L.; Romano, P.; Passacantando, M. Contact resistance and channel conductance of graphene field-effect transistors under low-energy electron irradiation. Nanomaterials 2016 6 (11), 206, doi: 10.3390/nano6110206.

19. Urban, F.; Lupina, G.; Grillo, A.; Martucciello, N.; Di Bartolomeo, A. Temperature and gate effects on contact resistance and mobility in graphene transistors by TLM and Y-function methods. arXiv 2019, arXiv:1912.04623.

20. Di Bartolomeo, A.; Genovese, L.; Giubileo, F.; Iemmo, L.; Luongo, G.; Foller, T.; Schleberger, M. Hysteresis in the transfer characteristics of MoS 2 transistors. 2D Mater. 2017, 5, 015014, doi:10.1088/2053-1583/aa91a7.

21. Qi, L.; Wang, Y.; Shen, L.; Wu, Y. Chemisorption-induced $n$-doping of MoS 2 by oxygen. Appl. Phys. Lett. 2016, 108, 063103, doi:10.1063/1.4941551.

22. Cho, K.; Kim, T.-Y.; Park, W.; Park, J.; Kim, D.; Jang, J.; Jeong, H.; Hong, S.; Lee, T. Gate-bias stress-dependent photoconductive characteristics of multi-layer MoS 2 field-effect transistors. Nanotechnology 2014, 25, 155201, doi:10.1088/0957-4484/25/15/155201.

23. Bao, W.; Cai, X.; Kim, D.; Sridhara, K.; Fuhrer, M.S. High mobility ambipolar MoS 2 field-effect transistors: Substrate and dielectric effects. Appl. Phys. Lett. 2013, 102, 042104, doi:10.1063/1.4789365.

24. Radisavljevic, B.; Kis, A. Mobility engineering and a metal-insulator transition in monolayer MoS2. Nat. Mater. 2013, 12, 815820, doi:10.1038/nmat3687. 
25. Urban, F.; Giubileo, F.; Grillo, A.; Iemmo, L.; Luongo, G.; Passacantando, M.; Foller, T.; Madauß, L.; Pollmann, E.; Geller, M.P.; et al. Gas dependent hysteresis in MoS 2 field effect transistors. 2D Mater. 2019, 6, 045049, doi:10.1088/2053-1583/ab4020.

26. Di Bartolomeo, A.; Urban, F.; Passacantando, M.; McEvoy, N.; Peters, L.; Iemmo, L.; Luongo, G.; Romeo, F.; Giubileo, F. A WSe2 vertical field emission transistor. Nanoscale 2019, 11, 1538-1548, doi:10.1039/C8NR09068H.

27. Fowler, R.H.; Nordheim, L. Electron Emission in Intense Electric Fields. Proc. R. Soc. A Math. Phys. Eng. Sci. 1928, 119, 173-181, doi:10.1098/rspa.1928.0091. 